\title{
Caracterização de mulheres que não procuraram o resultado do exame Papanicolaou em uma Unidade Básica de Saúde
}

\author{
Lyvia Maria Fernandes $^{1}{ }^{\oplus}$, Francisco Assis Cavalcante Junior ${ }^{2} @$, Rogéria \\ Mônica Seixas Xavier de Abreu ${ }^{3}$ ๑) Laurita da Silva Cartaxo ${ }^{4}$ @ \& Marilena \\ Maria de Souza ${ }^{4}$ ()
}

(1) Universidade Federal de Campina Grande, Centro de Formação de Professores, Unidade Acadêmica de Ciências da Vida, Rua Sérgio Moreira de Figueiredo, Casas Populares 58900-000, Cajazeiras, Paraíba, Brasil. E-mail: lyvia_fernandes@outlook.com

(2) Universidade Federal de Campina Grande, Centro de Formação de Professores, Unidade Acadêmica de Enfermagem, Rua Sérgio Moreira de Figueiredo, Casas Populares 58900-000, Cajazeiras, Paraíba, Brasil. E-mail: junior_cz228@hotmail.com

(3) Universidade Regional do Cariri - campus Iguatu, Avenida Dário Rabêlo, Santo Antônio 63500-000, Iguatu, Ceará, Brasil. E-mail: seixasxavier@hotmail.com

(4) Universidade Federal de Campina Grande, Centro de Formação de Professores, Escola Técnica de Saúde de Cajazeiras, Rua Sérgio Moreira de Figueiredo, Casas Populares, 58900-000, Cajazeiras, Paraíba, Brasil. E-mail: lauritascartaxo@gmail.com, marilenacarolino@gmail.com

Fernandes L.M., Cavalcante Junior F.A., Abreu R.M.S.X., Cartaxo L.S. \& Souza M.M. (2021) Caracterização de mulheres que não procuraram o resultado do exame Papanicolaou em uma Unidade Básica de Saúde. Pesquisa e Ensino em Ciências Exatas e da Natureza, 5(edição especial 1): e1641. http://dx.doi.org/10.29215/pecen.v5i0.1641

Responsabilidade editorial: Carla Heloísa A. de Figueiredo. Recebido: 02 dezembro 2020. Aceito: 04 janeiro 2021. Publicado: 03 fevereiro 2021.

Resumo: Este trabalho objetivou caracterizar as mulheres que não procuraram o resultado do exame Papanicolaou em uma Unidade Básica de Saúde no município de Cajazeiras - Paraíba, correlacionando os resultados com a idade, início da atividade sexual (IAS) e o número de parceiros, conforme resultados positivos e negativos para Infecções Sexualmente Transmissíveis (IST). A amostra foi de 113 exames, coletados no período de janeiro de 2010 a dezembro de 2015 . Verificou-se que a faixa etária que prevaleceu dentre os laudos positivos foi de 14-24 e de 36-46 anos, com 8 casos em cada; nos laudos negativos e mais um caso de alteração celular possivelmente não neoplásica, a faixa etária que prevaleceu foi de 25-35, com 25 casos. A média de idade do IAS em mulheres com resultados positivos e negativos foi $18.13( \pm 3.11)$ anos e $19.49( \pm 4.57)$ anos, respectivamente. Quanto ao número de parceiros sexuais, nos resultados positivos a média foi $2.03( \pm 1.37)$ parceiros, e nos negativos foi $1.72( \pm 1.21)$. Logo, o perfil de mulheres com idade inferior a 24 anos, IAS precoce e maior número de parceiros teve maior frequência nos laudos positivos para IST, estabelecendo uma relação entre estes fatores e a sua prevalência.

Palavras chave: Papanicolau, IST, câncer de colo uterino, medicina preventiva.

Characterization of women who did not seek the result of the paper examination in a basic health unit

Abstract: This study aimed to characterize women who did not seek the results of the Pap smear in a Basic Health Unit in the municipality of Cajazeiras - Paraíba, correlating the results with age, the onset of sexual activity and the number of partners, according to positive and negative results for Sexually Transmitted Infections (STIs). The sample consisted of 113 exams, collected from January 2010 to 
December 2015. It was found that the age group that prevailed among the positive reports was 14-24 and 36-46 years, with 8 cases in each; in the negative reports and another case of cellular alteration possibly not neoplastic, the age group that prevailed was 25-35, with 25 cases. The average age of onset of sexual activity in women with positive and negative results was $18.13( \pm 3.11)$ years and $19.49( \pm 4.57)$ years, respectively. As for the number of sexual partners, in the positive results, the average was $2.03( \pm 1.37)$ partners, and in the negative results, it was $1.72( \pm 1.21)$. Therefore, the profile of women under the age of 24 , beginning of an early sexual activity and a greater number of partners had a higher frequency in positive reports for STIs, establishing a relationship between these factors and their prevalence.

Key words: Pap smear, STIs, uterine cervical neoplasms, preventive medicine.

\section{Introdução}

Segundo a estimativa realizada pelo BRASIL (2020), o número de casos novos de câncer do colo do útero esperados para o Brasil, para cada ano do triênio 2020-2022, será de 16.590, com um risco estimado de 15.43 casos a cada 100 mil mulheres, denotando a alta frequência que essa condição clínica afeta a população feminina brasileira (BRASIL 2020).

O exame Papanicolaou consiste no esfregaço de células oriundas da ectocérvice e da endocérvice, que são extraídas por raspagem do colo do útero. $O$ resultado de tal exame auxilia $o$ programa de prevenção ao câncer do colo do útero e é o melhor método para identificar lesões em fase inicial e efetuar o diagnóstico da enfermidade. No Brasil é recomendado o rastreamento do câncer do colo do útero por esse exame no público alvo de mulheres entre 25 e 64 anos, que já iniciaram a vida sexual. $\mathrm{O}$ exame pode ser realizado nas unidades básicas de saúde por profissionais qualificados, com intervalo indicado entre os exames de três anos, após dois exames anuais negativos para neoplasia do colo uterino (BRASIL 2016).

Além disso, o exame Papanicolaou também adverte caso a paciente apresente alguma Infecção Sexualmente Transmissível (IST) que necessite de tratamento. A infecção pelo papilomavírus humano (HPV) causa 530.000 casos de câncer de colo uterino e 275.000 mortes por essa doença/ano. Alguns dos fatores determinantes são a influência sociocultural no comportamento sexual, a pobreza, as desigualdades de gênero, os conflitos sociais, entre outros. Para tanto, a atuação dos gestores da saúde deve aproveitar todas as oportunidades para assegurar insumos necessários ao desenvolvimento das ações de controle, dentro e fora dos serviços de saúde (BRASIL 2015).

Outrossim, a IST encontra-se entre os problemas de saúde pública mais comuns, constituindo a segunda causa de morbidade em mulheres nos países em desenvolvimento, exigindo atenção especial das políticas públicas de saúde, devido a sua alta taxa de prevalência e dificuldade na implementação de ações de diagnóstico precoce (Luppi et al. 2011).

A baixa idade das primeiras relações sexuais, a variabilidade de parceiros, o não uso de preservativo e o uso de drogas ilícitas é caracterizado como fatores de risco às ISTs. Do ponto de vista biológico, o epitélio cilíndrico do colo do útero na adolescência se encontra mais exposto e tanto as clamídeas como os gonococos têm predileção por este tecido. No âmbito psicológico, essa é uma fase de definição da identidade sexual com experimentação e variabilidade de parceiros, o que caracteriza uma vulnerabilidade maior (Beserra et al. 2008).

Dentre as razões para o não retorno das mulheres para receber o resultado do exame Papanicolaou, destacam-se motivos pessoais, relacionados ao esquecimento, e a falta de orientação clara sobre a importância do retorno para a validade da coleta do exame pelo serviço de saúde, além da dificuldade em conseguir novo atendimento, quando, por algum motivo, a mulher não pode comparecer na data prevista devido à longa espera para o atendimento ou marcação de uma consulta (Greenwood et al. 2006).

Diante de sua importância no contexto atual, buscou-se avaliar a IST por meio dos resultados do exame Papanicolaou não retirados pelas mulheres em uma Unidade Básica de Saúde (UBS) e correlacionar os resultados do exame com Início da Atividade Sexual (IAS) e o número de parceiros, conforme resultados positivos e negativos para IST. 


\section{Material e Métodos}

Trata-se do recorte de uma pesquisa intitulada "Avaliação dos resultados do exame papanicolauou não procurados por mulheres de uma Unidade Básica de Saúde”, de caráter documental, exploratória e quantitativa.

Tal pesquisa foi realizada na Unidade de Saúde Casas Populares/PAPS, no bairro Casas Populares em Cajazeiras - Paraíba, Brasil. O critério de escolha desta unidade foi o grande número de exames Papanicolaou armazenados para estudo. A Unidade de Saúde Casas Populares foi a única unidade local a arquivar o resultado dos exames de pacientes por um longo período de tempo. A população ou universo de dados é o conjunto de seres animados ou inanimados que detém uma ou mais características em comum (Lakatos \& Marconi 2010).

A população deste estudo foi composta por todos os exames de Papanicolaou realizados, porém não retirados pelas pacientes, no período de janeiro de 2010 a dezembro de 2015. Para tanto, a amostra foi composta por 113 resultados dos exames que obedeceram aos critérios de inclusão, a saber: exames que não foram retirados no período de janeiro de 2010 a dezembro de 2015 e os que continham o registro do resultado até a data da coleta dos dados. Os dados do livro de registro com os resultados dos exames, mas sem conter todas as informações que atendessem aos objetivos do estudo foram excluídos.

A coleta de dados ocorreu no mês de setembro de 2016, depois de ter sido estabelecido um contato inicial com a Unidade de Saúde da Família (USF). Os dados foram coletados nos livros de registro dos resultados dos exames de Papanicolaou e nos arquivos da Unidade de Saúde em sala reservada para manter o sigilo dos dados. Para um melhor manuseio e obtenção de informações dos documentos do estudo, utilizou-se uma planilha do Microsoft Excel como instrumento de coleta de dados. Os dados foram tabulados no software Microsoft Office Excel 2013 para ambiente Windows. A análise estatística foi realizada para verificar as medidas de tendência central das variáveis idade, IAS, número de parceiros e frequência das IST.

Além disso, realizou-se uma correlação de Pearson entre as variáveis IAS e o número de parceiros dos grupos com resultados positivos e negativos para IST, sendo utilizado o software Statistica 7.0 da Statsoft para ambiente Windows. Uma medida de associação de variáveis foi feita, calculando o coeficiente de correlação de Pearson. Esse coeficiente, o $\rho$ de Pearson, mede a intensidade da relação entre variáveis ordinais não sendo sensível a assimetrias na distribuição, nem à presença de “outliers”, não exigindo, portanto, que os dados provenham de duas populações normais. O coeficiente $\rho$ de Pearson varia entre -1 e 1. Quanto mais próximo estiver destes extremos, maior será a associação entre as variáveis. O sinal negativo da correlação significa que as variáveis variam em sentido contrário, isto é, as categorias mais elevadas de uma variável estão associadas a categorias mais baixas da outra variável e vice e versa para o sinal positivo.

A pesquisa foi aprovada pelo Comitê de Ética em Pesquisa do CFP/UFCG parecer número 1.478167, e CAAE: 53864316.3.00005575, seguindo as exigências éticas da Resolução 466/12.

\section{Resultados e Discussão}

Cento e treze (113) resultados de laudos do exame de Papanicolaou não retirados pelas mulheres foram identificados, no livro de registro da UBS escolhido para o estudo. Todos os registros empregados nesta pesquisa encontraram-se preenchidos corretamente e com resultado de amostras aceitáveis. Dos 113 resultados analisados, 30 (26\%) tiveram resultados positivos, sendo que apenas um exame Papanicolau apresentou resultado positivo para duas IST. Já os resultados negativos para IST foram encontrados em $82(73 \%)$ laudos, ressaltando que houve mais $1(1 \%)$ resultado de alteração celular possivelmente não neoplásica.

$\mathrm{Na}$ Figura 1, os resultados demonstram que a faixa etária que prevaleceu dentre os 30 (100\%) resultados positivos para IST, respectivamente, foi a de 14-24 com 8 (27\%) casos e a de 36-46 com 8 (27\%) casos; seguidos de 25-35 com 7 (22\%) casos; 47-57 com 5 (17\%) casos e 5868 com $2(7 \%)$ casos. Já entre os $82(99 \%)$ resultados negativos para IST, e mais 1 (1\%) caso de 
alteração celular possivelmente não neoplásica, a faixa etária que prevaleceu foi a de $25-35$ com $25(30 \%)$ casos; seguida de 36-46 com 22 (27\%) casos; 47-57 com 12 (14\%) casos; 58-68 com 10 (12\%) casos; $14-24$ com 9 (11\%) casos; $69-79$ com 3 (4\%) casos e $80-85$ com 2 (2\%) casos.

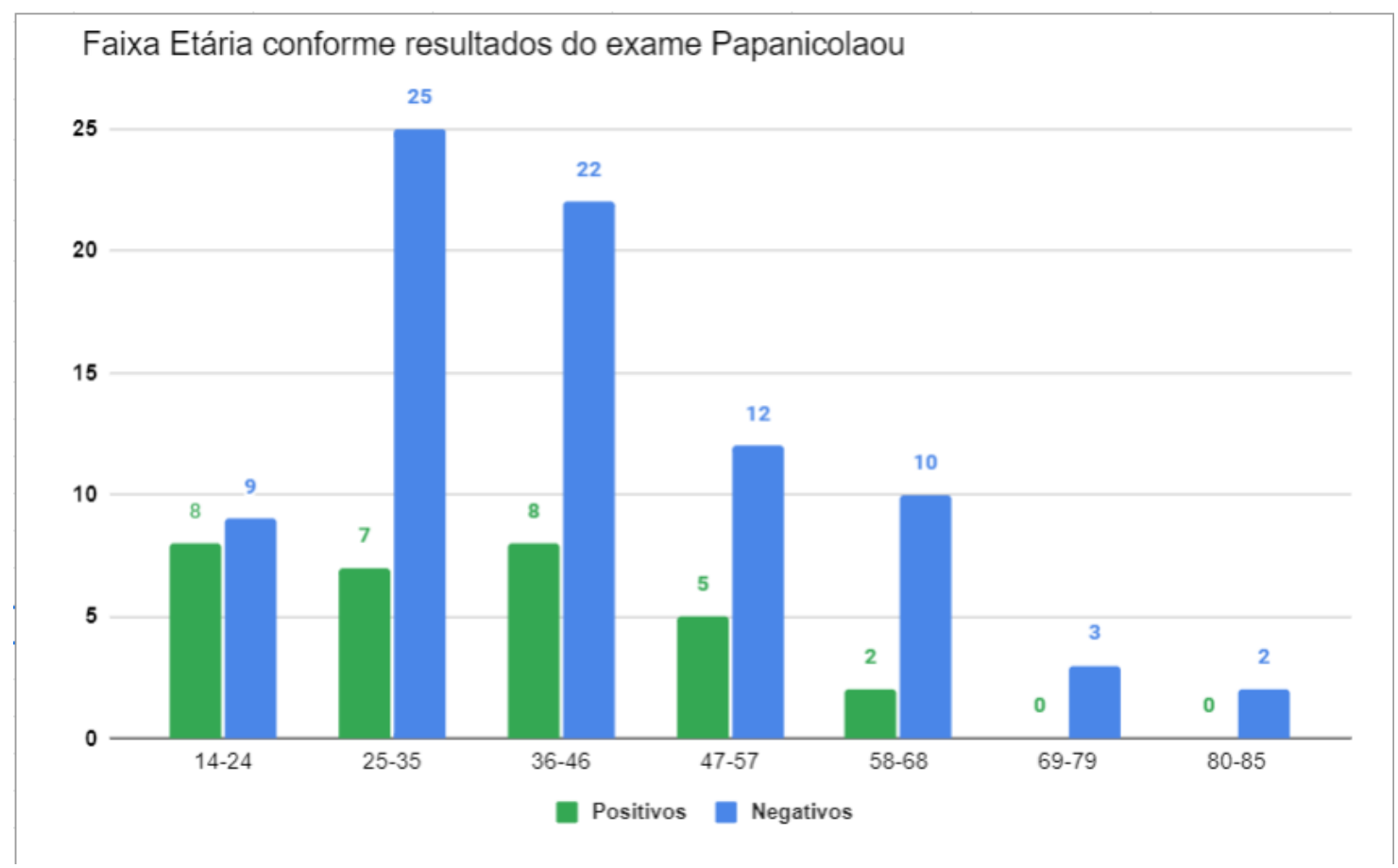

Figura 1. Distribuição das mulheres identificadas no estudo por faixa etária e conforme resultados na cidade de Cajazeiras (PB) entre 2010 e 2015 que não procuraram os resultados do exame Papanicolaou.

Conforme o estudo realizado por Luppi et al. (2011), sobre diagnóstico precoce e os fatores associados às infecções sexualmente transmissíveis em mulheres atendidas na atenção primária, realizada na área central da região metropolitana de São Paulo - SP, obteve-se $22 \%$ de prevalência de IST em mulheres de até 20 anos. A idade mínima obteve $27 \%$ de prevalência de IST em mulheres de 14-24 anos. Para os autores referenciados, as jovens dispõem de maior probabilidade de possuírem número de parceiros sexuais superior, relação desprevenida e parceiros sexuais com significativa exposição a riscos, o que dá ênfase a vulnerabilidade desta faixa etária para contrair IST.

É importante mencionar que para as mulheres na faixa etária de 14-24 anos não é dada preferência na realização do exame Papanicolaou, mas sim as mulheres de 25-64 anos, já que entre esta idade, conforme BRASIL (2016) existe maior incidência de lesões de alto grau susceptíveis a tratamento, evitando sua evolução para o câncer. Antes dos 25 anos, predominam os casos de HPV juntamente com as lesões de baixo grau, que muitas vezes retrocedem espontaneamente, fazendo necessário apenas o acompanhamento de acordo com as indicações clínicas.

Na Tabela 1, os resultados mostram que nas mulheres com resultados positivos para IST a idade foi de 17 a 68 anos, a média foi de $36.26( \pm 13.84)$ anos. Com relação aos resultados negativos para IST, a idade foi de 14 a 85 anos, a média foi de $41.71( \pm 16.43)$ anos.

Pode-se notar, no que diz respeito a idade mínima e máxima das mulheres observadas nos laudos avaliados, sendo 14 e 85 anos, respectivamente, uma proximidade com os dados da pesquisa de Pinheiro et al. (2014), "Análise dos exames colpocitológicos não retirados por pacientes entre 2007 e 2009: estudo documental” realizada em um Centro de Saúde da Família na periferia de Fortaleza - CE, onde a idade foi de 10 a 83 anos. 
Tabela 1. Medidas de tendência central e idade das mulheres que não procuraram os resultados do exame Papanicolaou na cidade de Cajazeiras (PB) entre 2010 e 2015.

\begin{tabular}{ccccc}
\hline Características Pessoais & \multicolumn{3}{c}{ POSITIVO } \\
\hline \multirow{2}{*}{ Idade } & $\mathrm{N}$ & Mínima & Média \pm Desvio Padrão & Máxima \\
& 30 & 17 & $36.26 \pm 13.84$ & 68 \\
\hline \multirow{2}{*}{ Idade } & $\mathrm{N}$ & Mínima & Média \pm Desvio Padrão & Máxima \\
& 83 & 14 & $41.71 \pm 16.43$ & 85 \\
\hline
\end{tabular}

Na Tabela 2, constatou-se que as mulheres com resultados positivos para IST, a idade de início da atividade sexual foi de 12 a 28 anos, com média de 18.13 ( $₫ 3.11)$ anos. Com relação aos resultados negativos para IST e início da atividade sexual, a idade foi de 12 a 38 anos, com média de $19.49( \pm 4.57)$ anos.

Tabela 2. Medidas de tendência central e IAS das mulheres que não procuraram os resultados do exame Papanicolaou na cidade de Cajazeiras (PB) entre 2010 e 2015.

\begin{tabular}{ccccc}
\hline Características Pessoais & \multicolumn{4}{c}{ POSITIVO } \\
\hline \multirow{2}{*}{ IAS } & $\mathrm{N}$ & Mínima & Média \pm Desvio Padrão & Máxima \\
& 30 & 12 & NEGATIVO & 28 \\
\hline \multirow{2}{*}{ IAS } & $\mathrm{N}$ & Mínima & Média \pm Desvio Padrão & Máxima \\
& & & & \\
& & 12 & $19.49 \pm 4.57$ & 38 \\
\hline
\end{tabular}

Podemos constatar também que os resultados da média da idade do início da atividade sexual, positivos $(18.13 \pm 3.11)$ e negativos $(19.49 \pm 4.57)$ demonstram que as mulheres que iniciam a atividade sexual mais precocemente estão mais susceptíveis a contrair uma IST. Esse resultado corrobora com a pesquisa de Santos et al. (2007), intitulada “Alterações cérvico-uterinas em mulheres atendidas em uma unidade básica de saúde no município de Campinas - SP” quando afirmam que as mulheres com início precoce da atividade sexual encontram-se mais propensas a adquirir IST, especialmente o HPV.

O estudo realizado por Araújo et al. (2012), sobre os "fatores de risco para infecção por HIV em adolescentes”, desenvolvido no norte de Teresina - PI, afirmam que as IST estão acontecendo cada vez mais cedo, expondo, principalmente os adolescentes a essas infecções.

$\mathrm{Na}$ Tabela 3, em relação ao número de parceiros, os resultados positivos e negativos para IST variou de 1 a 7 , com média, no primeiro caso de $2.03( \pm 1.37)$ e no segundo caso de 1.72 $( \pm 1.21)$ parceiros. Luppi et al. (2011) na pesquisa "Diagnóstico precoce e os fatores associados às infecções sexualmente transmissíveis em mulheres atendidas na atenção primária” realizada na área central da região metropolitana de São Paulo - SP, com mulheres de 18 a 40 anos, mostraram que os fatores de risco ligados a presença de, pelo menos, uma das três IST procuradas no estudo foram: ser jovem, maior número de parceiros sexuais na vida, cor de pele ou etnia indígena e auto percepção de risco; corroborando, desta forma, com esse estudo no que diz respeito ao número de parceiros, já que nos laudos com resultados positivos para IST nessa pesquisa a média foi superior aos laudos com resultados negativos. Além disso, o estudo realizado por Santos et al. (2014), sobre "Características sociodemográficas e risco para doenças sexualmente transmissíveis entre mulheres atendidas na atenção básica” realizado em São Cristóvão - SE, afirmam que a multiplicidade de parceiros sexuais torna as mulheres mais susceptíveis as IST. 
Tabela 3. Medidas de tendência central e número de parceiros de mulheres que não procuraram os resultados do exame Papanicolaou na cidade de Cajazeiras (PB) entre 2010 e 2015.

\begin{tabular}{|c|c|c|c|c|}
\hline Características Pessoais & & & POSITIVO & \\
\hline \multirow{4}{*}{ Parceiros } & $\mathrm{N}$ & Mínima & Média \pm Desvio Padrão & Máxima \\
\hline & & & & \\
\hline & 30 & 1 & $2.03 \pm 1.37$ & 7 \\
\hline & & & NEGATIVO & \\
\hline \multirow{3}{*}{ Parceiros } & $\mathrm{N}$ & Mínima & Média \pm Desvio Padrão & Máxima \\
\hline & & & & \\
\hline & 83 & 1 & $1.72 \pm 1.21$ & 7 \\
\hline
\end{tabular}

A associação utilizando a correlação de Pearson mostrou que a correlação entre IAS e o número de parceiros foi fraca (0.14) para as mulheres com laudos de resultados positivos, pois está próxima de 0 (zero), conforme enfatizada pela dispersão dos pontos (Figura 2). Outrossim, a Figura 3 mostra a correlação entre IAS e o número de parceiros sendo quase nula (0.018) para com as mulheres representadas pelos laudos com resultados negativos, pois está próxima de 0 (zero), enfatizada pelos pontos que encontram-se dispersos no gráfico. A pesquisa de Hugo et al. (2011) intitulada "Fatores associados à idade da primeira relação sexual em jovens: estudo de base populacional”, realizada com jovens residentes na zona urbana da cidade de Pelotas - RS, relata não haver nenhuma relação estatística importante entre o IAS e o número de parceiros sexuais.

Notoriamente, para identificar a existência da correlação do IAS e número de parceiros para as mulheres com laudos do exame Papanicolaou positivos para IST, observou-se um certo deslocamento à direita, justificando-se pelo fato de que as mulheres com resultados positivos para IST, que tiveram IAS antes dos 15 anos, possuíam um número de parceiros elevados; em seguida pelas mulheres com IAS até 20 anos. Contudo, a amostra pertencente aos resultados negativos apresentou maior número de parceiros nas mulheres com IAS após os 15 anos; em seguida pelas mulheres que IAS com 25 anos.

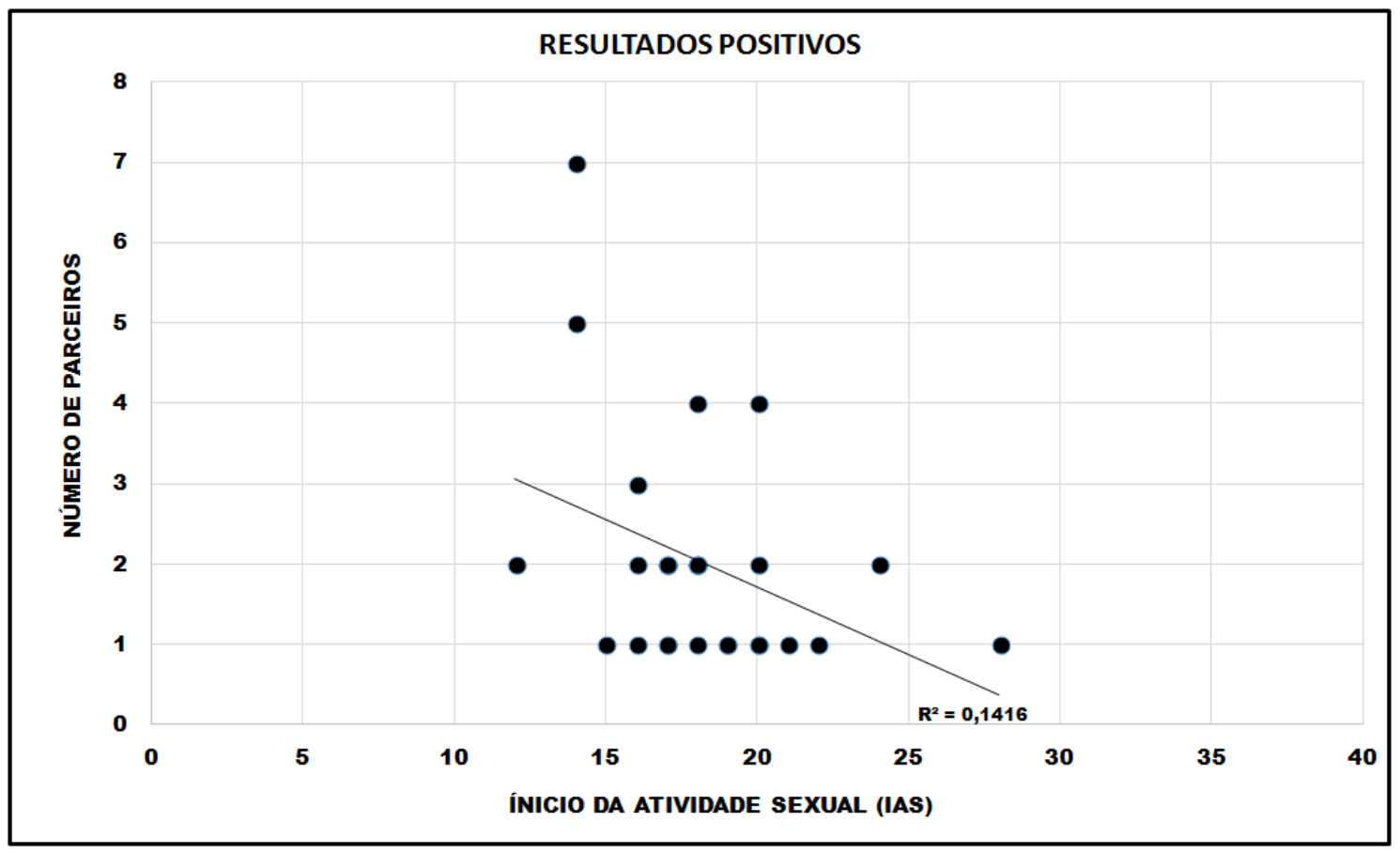

Figura 2. Correlação entre IAS e número de parceiros no grupo com resultados positivos. 


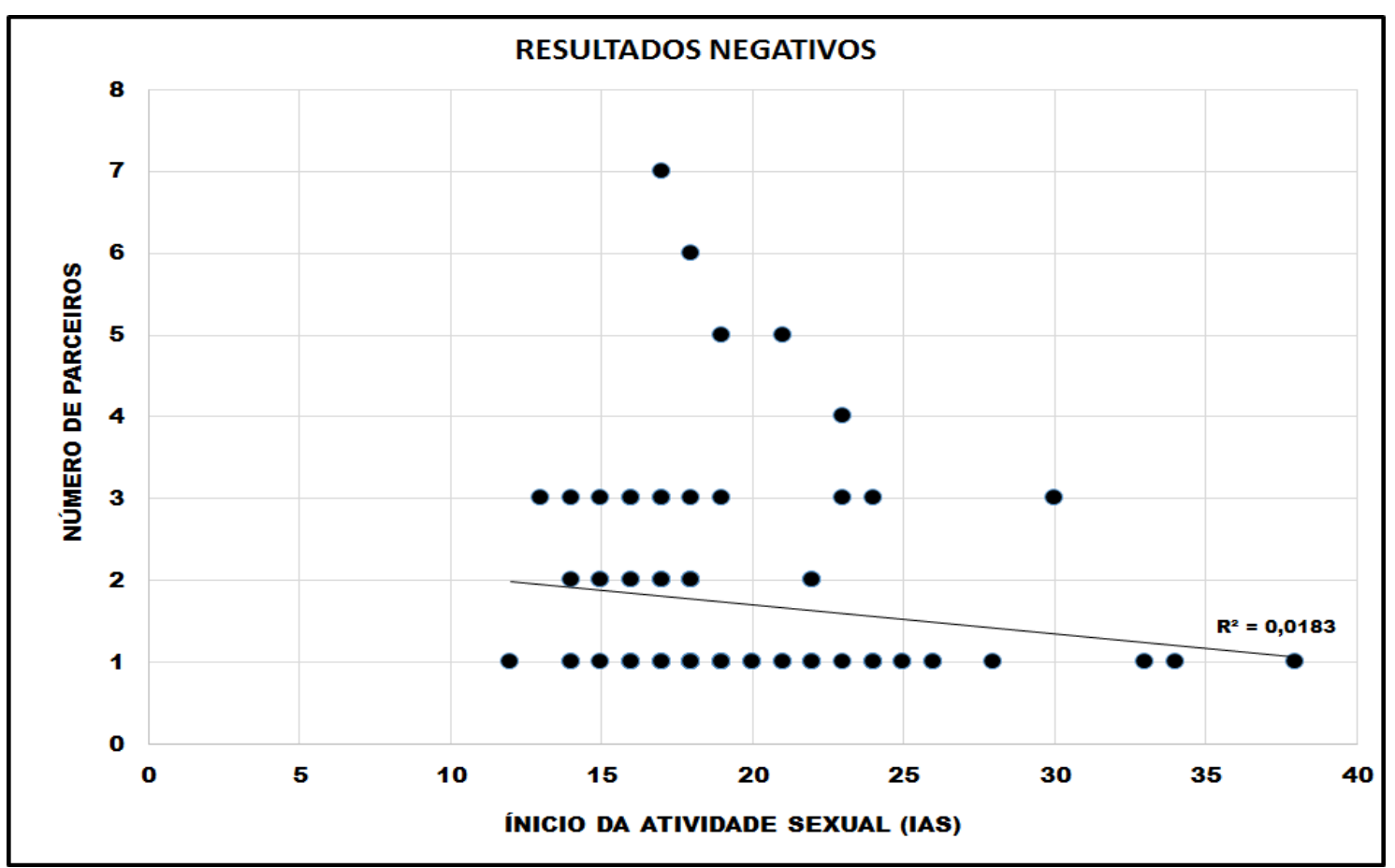

Figura 3. Correlação entre IAS e número de parceiros no grupo com resultados negativos.

\section{Conclusões}

Infere-se que houve um número acentuado de mulheres que não retornaram a UBS para recolher os laudos e que apresentaram IST. Dentre elas, o perfil que possuía idade inferior a 24 anos, início da atividade sexual precoce e maior número de parceiros, tornou-se mais presente nos laudos com resultados positivos para IST, desta forma, estabelecendo uma relação entre esses fatores e a sua prevalência na população estudada.

Para tanto, a correlação entre IAS e o número de parceiros foi fraca (0.14) para as mulheres com laudos de resultados positivos, sendo que foi quase nula (0.018) para com as mulheres representadas pelos laudos com resultados negativos.

Dessa forma, apesar dos resultados serem um importante acervo de dados para subsidiar intervenções qualificadas e efetivas, capazes de impactar na incidência dessas doenças nesses subgrupos vulneráveis, é necessário realizar políticas de rastreamento pelo serviço de saúde, principalmente direcionado às mulheres jovens, a fim de utilizar estratégias que venham a contribuir na redução do número de laudos não retirados por elas e correto seguimento médico.

As limitações deste trabalho incluem o tamanho modesto e a natureza única da amostra (uma UBS), o que limita os resultados a respeito de outras mulheres em condições similares que não foram avaliadas. No entanto, o alto número encontrado de mulheres que não retornaram à UBS e que, além disso, possuíam alguma IST demonstra como esse problema é pertinente dentro do contexto de saúde pública e vigilância epidemiológica, necessitando de mais trabalhos e estratégias para o correto manejo desses casos.

\section{Referências}

Araújo T.M.E., Monteiro C.F.S., Mesquita G.V., Alves E.L.M., Carvalho K.M. \& Monteiro R.M. (2012) Fatores de Risco para Infecção por HIV em Adolescentes. Revista Enfermagem UERJ, 20(2): 242-247.

Beserra E.P., Pinheiro P.N.C., Alves M.D.S. \& Barroso M.G.T. (2008) Adolescência e vulnerabilidade às doenças sexualmente transmissíveis: uma pesquisa documental. Jornal Brasileiro de Doenças Sexualmente Transmissíveis, 20(1): 32-35. 
BRASIL (2015) Ministério da Saúde. Secretaria de Ciência, Tecnologia e Insumos Estratégicos. Protocolo Clínico e Diretrizes Terapêuticas Infecções Sexualmente Transmissíveis. Brasília: Ministério da Saúde. 122 p.

BRASIL (2016) Instituto Nacional de Câncer José Alencar Gomes da Silva. Coordenação de Prevenção e Vigilância. Divisão de Deteç̧ão Precoce e Apoio à Organização de Rede. Diretrizes brasileiras para o rastreamento do câncer do colo do útero. $2^{\circ}$ edição. Rio de Janeiro: INCA. 114 p.

BRASIL (2020) Ministério da Saúde. Secretaria de Atenção à Saúde. Instituto Nacional de Câncer. Coordenação de Prevenção e Vigilância de Câncer. Estimativa 2020: incidência de câncer no Brasil. Rio de Janeiro: INCA. 122 p.

Greenwood S.A., Machado M.F.A.S. \& Sampaio N.M.V. (2006) Motivos que levam mulheres a não retornarem para receber o resultado de exame Papanicolau. Revista Latino-Americana de Enfermagem, 14(4): 503-509.

Hugo T.D.O., Maier V.T., Jansen K., Rodrigues C.E.G., Cruzeiro A.L.S., Ores L.C., Pinheiro R.T., Silva R. \& Souza L.D.M. (2011) Fatores Associados à Idade da Primeira Relação Sexual em Jovens: estudo de base populacional. Cadernos de Saúde Pública, 27(11): 2207-2214.

Lakatos E.M. \& Marconi M.A. (2010) Fundamentos de Metodologia Cientifica. $7^{\circ}$ edição. São Paulo: Atlas. 320 p.

Luppi C.G., Oliveira R.L.S., Veras M.A., Lippman S.A., Jones H., Jesus C.H., Pinho A.A., Ribeiro M.C. \& Caiaffa-Filho H. (2011) Diagnóstico precoce e os fatores associados às infecções sexualmente transmissíveis em mulheres atendidas na atenção primária. Revista Brasileira de Epidemiologia, 14(3): 467-477. https://doi.org/10.1590/S1415-790X2011000300011

Pinheiro M.A.C.F., Vasconcelos C.T.M., Vasconcelos Neto J.A., Cunha D.F.F. \& Pinheiro A.K.B. (2014) Análise dos exames colpocitológicos não retirados por pacientes entre 2007-2009: estudo documental. Online Brazilian Journal of Nursing, 13(1): 46-52.

Santos J.O., Silva S.R., Santos C.F., Araújo M.C.S. \& Bueno S.D. (2007) Alterações Cérvico-uterinas em Mulheres Atendidas em uma Unidade Básica de Saúde no Município de Campinas-SP. Revista Mineira de Enfermagem, 11(4): 439-445.

Santos L.V., Inagaki A.D.M., Abud A.C.F., Oliveira J.K.A., Ribeiro C.J.N. \& Oliveira M.I.A. (2014) Características Sociodemográficas e Risco para Doenças Sexualmente Transmissíveis entre Mulheres Atendidas na Atenção Básica. Revista Enfermagem UERJ, 22(1): 111-115. 